Contributions to Game Theory and Management, XIV, 91-102

\title{
Equilibrium in Generalized Stackelberg Game with Arbitrary Memory and Planning Horizon of Players
}

\author{
Denis Fedyanin \\ Institute of Control Sciences of Russian Academy of Sciences, 65 Profsoyuznaya street, \\ Moscow 117997, Russian Federation, \\ HSE University, 20 Myasnitskaya ulitsa, Moscow 101000, Russian Federation \\ E-mail: dfedyanin@inbox.ru
}

\begin{abstract}
The paper investigates game-theoretical properties of a model of production dynamics on markets with wrong expectations of most producers about the existence of a market. It might be a market of electrocars, green energy space flights, paper books, theaters, oil energy, etc. The foundation of a game-theoretical model is a special method for the generating of an epistemic model from observations. The method is based on a generalization of a classic model from the theory of mind and an idea of an observation model is very similar to the model of moving average. We focused on periodic solutions and introduced a control model for them. The control problem in the model is an optimization problem for parameters of induced parametric equilibrium in the game. The influence of the initial conditions on the overall dynamics was modeled for some examples.
\end{abstract}

Keywords: epistemic models, theory of mind, Cournot competition, evolution games.

\section{Introduction}

We consider a dynamic model of market production with predictions and memory. The model might be applied to various settings. Among other examples, this model describes the periodic market oscillations when the product expected lifetime is given, and the market believes that this market will be eliminated at a fixed horizon. It does not mean that the actual lifetime of products or market lifetime is given. We focus on actions of the market that depend on the beliefs of actors of this market. There is a model where the market can use current data to update beliefs, but our model does not use these types of updates. Examples of products where the horizon for planning is constant but the market rest lifetime exceed this horizon are Fusion power, non-green energy supplies, not-Bitcoin money.

Our approach might be an example of evolution games where we have several players with limited scope and horizon, but they have the same types and always choose the same strategies. It can also be used if it is possible to aggregate all players into one, which can be called market also. Thus, we have a game of several players which are market at different moments and with the individual utility. It corresponds to the market in developing countries where their interest guides producers because of the uncertain future of the market and unpredictable owners of the production companies. So the market tries to choose a production value, considering that some products are already produced, and customers might want to buy products, not as market price as an effect can include future productions as shares have prices that depend on beliefs of product buyers and sellers.

We construct an epistemic model throughout the following section to show motivations and formal language to carefully define what agents believe in and

https://doi.org/10.21638/11701/spbu31.2021.08 
what agents think about what other agents believe and so on (Aumann, 1999; Novikov and Chkhartishvili, 2014). We generalized a model in another paper, but we would like to add examples and explain in more detail. This model will also catch some effects that we did not consider earlier.

Epistemic construction is indeed an informational structure (Fedyanin, 2018) of the game, and since we do not use logic to prove theorems but for incorporating results into a larger framework, use standard notation for explaining the model and some results. There are dynamic epistemic logic, epistemic logic, Bayes-Nash equilibriums and other tools to handle asymmetric knowledge.

\section{Epistemic model}

An epistemic model that is used in the paper generalizes the epistemic model for Anna and Sally (Byom and Mutlu, 2013; Wimmer and Perner, 1983). There are other investigations in this domain, see (Huck and Müller, 2000). Ann and Sally experiment shows that people tend to consider non-observable as non-happening (Wimmer and Perner, 1983). Let some actions happen in a room. People who are in the room know who is in the room, and actions have happened. Let there are pairs (action, time), and each agent has a subset of time $\operatorname{Pres}_{i}$, which an agent $i$ is present in the room. Let all happened actions are $S$. The generalization is the following. Let there are agents, there is a room, and there is a list of observations of events, actions. We consider that every observation perfectly identifies the action and is common knowledge for all players in a room. An important example of such observation is an observation that an agent is present in the room, which is common knowledge. Observation might form a controversial system because they are partial since not all agents are always in the room and might not observe some actions. We can check if every agent's observation is controversial or not, but we do not describe agents' behaviour.

\subsection{Example}

The following table illustrates the observation concept.

Table 1. Agent observations in the example

\begin{tabular}{|c|c|c|c|c|c|}
\hline Observed operation & Anna & Sally & John & Denis & Dave \\
\hline empty all boxes & In & In & In & Out & Out \\
\hline put red ball in box 1 & In & Out & In & In & In \\
\hline move red ball from box 1 to box 2 & In & Out & In & In & In \\
\hline put blue ball in box 2 & In & In & In & In & In \\
\hline move red ball from box 2 to box 1 & In & In & Out & In & Out \\
\hline move blue ball from box 2 to box 3 & In & In & Out & Out & Out \\
\hline
\end{tabular}

\section{Ann observations}

1. empty all boxes

2. put red ball in box 1

3. move red ball from box 1 to box 2

4. put blue ball in box 2

5. move red ball from box 2 to box 1

6. move blue ball from box 2 to box 3 
Sally observations

1. empty all boxes

2. put blue ball in box 2

3. move red ball from box 2 to box 1

4. move blue ball from box 2 to box 3

Denis observations from the Sally point of view

1. put blue ball in box 2

2. move red ball from box 2 to box 1

Dave observations from John point of view from Denis point of view from Sally point of view

1. put the blue ball in box 2

So the epistemic results are

- blue ball in box 3, red ball in box 1, no other balls in boxes for Ann

- inconsistency is for Sally. Sally did not know when the red ball was put in box 2 , but the blue ball in box three and the red ball is in box one now

- red ball is in box 1 , blue ball in box 2 , no other information about the content of boxes for the image of Denis

- blue ball in box 2, no other information about the content of boxes for the image of Dave

\subsection{General epistemic notation}

Let denote $B_{i} \alpha_{k}$ if an agent $i$ believes that observation $\alpha_{k}$ took place, $B_{i} B_{j} \alpha_{k}$ if an agent $i$ believes that an agent $j$ believes that an observation $\alpha_{k}$ took place. It might be described in terms of standard modal logic with modalities for every agent and might be temporal modalities,

$$
\varphi:=\alpha|\neg \varphi|(\varphi \wedge \psi) \mid B_{i} \varphi
$$

Here we use $\varphi$ and $\phi$ to denote a formula that describe observation and epistemic conclusions of the agents. The form of formula description should be read a formula is either an observation, or a negation of another formula, or boolean combination of two other formulas, or a belief of an agent about another formula.

It was suggested in the paper that if $S_{i}$ is the set of actions which have happened when an agent $i$ was in the room then

$$
B_{i_{1}} \ldots B_{i_{m}} \alpha_{i} \leftrightarrow\left(\alpha_{i} \in \cap_{k \in\left\{i_{1}, \ldots, i_{m}\right\}} S_{k}\right)
$$

The important consequence is that for any finite set $i_{1}, \ldots i_{k}$ if $k \geq n$ then there is a $m \leq n$ such that there is a finite set $j_{1}, \ldots, j_{m}$ that

$$
B_{i_{1}} \ldots B_{i_{k}} p_{i}=B_{j_{1}} \ldots B_{j_{m}} \alpha_{i}
$$

It works even if we have an formulas with operator $C \alpha_{i}$ of common believe

$$
C \alpha_{i}=\alpha_{i} \cap B_{i_{1}} \alpha_{i} \cap \ldots \cap B_{i_{1}} \ldots B_{i_{k}} \alpha_{i} \cap \ldots
$$




\subsection{Diagrams for an epistemic model of game}

Diagrams in this subsection show scopes which define epistemic models. We consider three critical cases: the periodic solution, the dynamic near the start of the game and the dynamic near-real end of the game. We have calculated some data as an example for the second and the third cases. Unfortunately, we cannot show the solution in analytical form because of the very sophisticated output. We comment on why periodic solutions are essential and mention some problems to compline solutions for all three cases into a complete solution.

$$
\ldots \leftarrow k \leftarrow \underbrace{(k+1) \leftarrow(k+2)}_{\text {memory }} \leftarrow \underbrace{(k+3)}_{\text {agent }} \leftarrow \underbrace{(k+4) \leftarrow(k+5)}_{\text {horizon }} \leftarrow \ldots
$$

Fig. 1. Structure of scope of an agent $k+3$

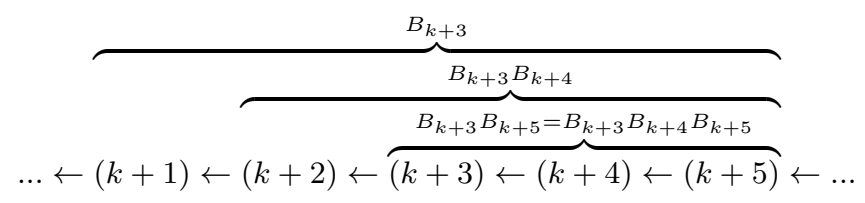

Fig. 2. A scope $B_{k+3}$ of agent $k+3$ and phantom agents in his mind in the middle of the game, if memory $h=2$ and prediction $p=2$. The agent is aware of two agents before him and two agents after. $B_{k+3} B_{k+4}$ shows what is in a scope of agent $k+4$ from a perspective of agent $k+3$.

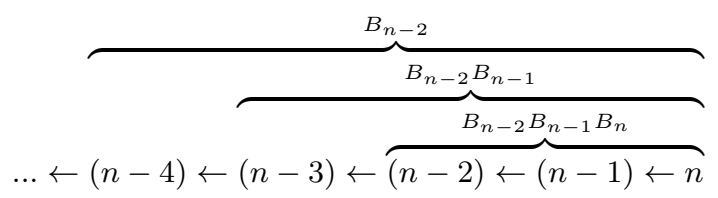

Fig. 3. A scope $B_{n-2}$ of agent $n-2$ and phantom agents in his mind near the end of the game, if memory $h=2$ and prediction $p \geq 2$. The agent is aware of two agents before him and more than one agent after. $B_{n-2} B_{n-1}$ shows what is in a scope of agent $n-2$ from a perspective of agent $n-3$. Unlike middle game the diagram is valid not only for $p=2$ but for any $p \geq 2$.

\section{Games}

Let $n$ agents choose their strategies in a given order. Every agent observes strategies of $h$ previous agents and believes that a game will stop after $p$ next agents chose 


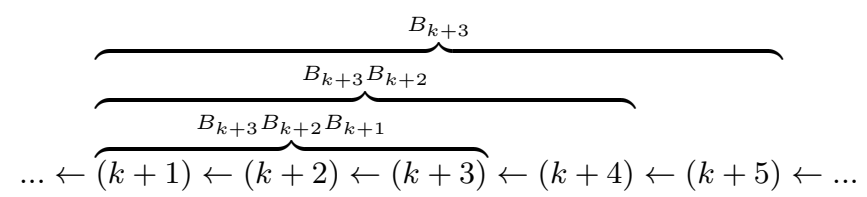

Fig. 4. A scope of agent $k+3$ and phantom agents in his mind in middle of the game, if memory $h=2$ and prediction $p=2$. The agent is aware of two agents before him and two agents after. $B_{k+3} B_{k+2}$ shows what is in a scope of agent $k+2$ from a perspective of agent $k+3$.

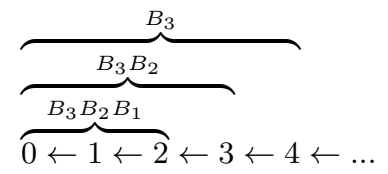

Fig. 5. A scope $B_{4}$ of agent 4 and phantom agents in his mind at the begining of the game, if memory $h \geq 3$ and prediction $p=1$. The agent is aware of two agents before him and more than one agent after. $B_{4} B_{3}$ shows what is in a scope of agent 3 from a perspective of agent 4 . Unlike middle game the diagram is valid not only for $h=3$ but for any $p \geq 3$.

their strategies. Statelbers's game (Li and Sethi, 2017) is such game, when $n=2$, $p=1, h=1$.

\subsection{Description}

The Cournot competition (Allaz and Vila, 1993) produces a system of linear equation. The solution depends on observing an agent's strategies, and the dependence is a linear function. Using the linear recursive methods, we can construct the analytical solution that is polynomial of power functions.

We suggest modifications: assign two parameters to each player - memory and planning horizon or prediction $\mathrm{p}$, built corresponding epistemic models for players and calculate equilibria.

The algorithm of decision for an agent is to look at the last known agent and predict its choice by the best response. This choice will depend on the strategy of the previous players. Thus previous players can influence. We use agents and players as synonyms.

The activity of agents is synchronized with a step generator. The game can be interpreted if the action's duration is $h$, the duration of the presence of an agent in the room is $p$, and a new agent comes into the room every step. It can also be interpreted as a cultural shift during the change of generations.

Let us consider a game with a given set of players fundamental positive strategies. A player's utility function depends only on the player's strategy and the sum of strategies of all players.

An example of a game with utility function and strategies is Cournot competition and one of the Tullock rent-seeking game versions. It is a well-known class of games. Furthermore, we modify the game of this class and focus on the modified 
game. We suggest three modifications. Following the Stackelberg competition model idea, we introduce ordering in deciding between players. We consider ordering as a parameter and will investigate the role of this parameter on players' decision and their utility functions (Rapoport, 1997; Abele, et al., 2004; Huck and Müller, 2000; Spiliotopoulou et al., 2019).

The second and third modification is an assignment of two types to each player. A player has memory and planning horizons. Memory is how many preceding players decisions the player can observe or remember. If a player makes her decision late enough and her memory is small enough, then the first player's strategy is to be neglected by the player. For the sake of simplicity, we assume that the player will behave as if the first player has chosen a zero strategy. The planning horizon is how far the player looks at the future and when the game will stop from her perspective.

There are opportunities to handle difficulties with players belief about other players planning horizon, similar to $k$-level epistemic models (Novikov and Chkhartishvili, 2014). However, we will avoid these difficulties in the leading case. We use the assumption that a player fixes her belief about when the game stop and when it has been started and consider it is accurate and shared knowledge (Aumann, 1999; Fedyanin, 2018; Novikov and Chkhartishvili, 2014). We found equilibrium for different general cases of the modified game and investigated these modes.

We can use the linear recursive method to find the solution if we have $h$ history, equal $r$ type, $p$ - prediction.

Then the general method is to predict the best action for the agent $j+p$. His action depends on the actions of all previous. The previous is a function of its previous and so on. Then we have the best response.

\subsection{Model of the game}

There is a set $N=1, \ldots, m$ of agents, each agent $i$ has type $(p, h)$. The actual utility function for the agent $i$ is $f_{i}$ and a utility function $F=\sum_{j \in N} x_{j}$ for the Principal. We consider a game $G=\langle N, X, f, I\rangle$, where $X=\left\{X_{i}=\{x\right.$ geq0 $\left.\}\right\}$ is a set of possible strategies for agents. Though we use games will keep reference to them as agents, not players. $I$ is an information structure of the game which has been described above in the epistemic section.

Though the approach is very general we will focus here on the Cournot competition to use sine results which we derived before.

$$
f_{i}=x_{i}\left(A-\sum_{j \in N} x_{j}\right)-\frac{x_{i}^{2}}{r_{i}} .
$$

There is an interpretation of parameters and variables for Cournout competition.

- $A$ is market volume,

$-x_{i}$ is production of the agent $i$,

- $\left(A-\sum_{j \in N} x_{j}\right)$ is a price for the products on the market,

- $x_{i}\left(A-\sum_{j \in N} x_{j}\right)$ is a multiplication of production and price and, thus, it is a profit

$-\frac{x_{i}^{2}}{r_{i}}$ is a cost function

$-r_{i}$ is a type of the agent. The larger type the more efficient is an agent. 
And agent $i+1$ chooses this strategy once right after an agent $i$. An agent 0 chooses his strategy first. However, an agent $i$ has another belief about his utility function. He believes that it is

$$
f_{i}=x_{i}\left(A-\sum_{j \in\{\min \{0, i-h\}, \ldots, i+p\}} x_{j}\right)-\frac{x_{i}^{2}}{r_{i}} .
$$

At the same time, he believes that other players play the corresponding other game. Let us denote $S_{i}=\{\min \{0, i-h\}, \ldots, i+p\}$. For any sequence of agents from $N$, say $j_{1}, \ldots, j_{m}$, the agent $j_{1}$ believes that an agent $j_{2}$ believes $\ldots$ the agent $j_{m}$ has a utility function

$$
f_{j_{m}}=x_{j_{m}}\left(A-\sum_{k \in \cap_{b \in j_{1}, \ldots j_{m} S_{b}}} x_{k}\right)-\frac{x_{j_{m}}^{2}}{r_{j_{m}}} .
$$

The difficulty is to handle all belief carefully to find an equilibrium.

\subsection{Method of equilibria calculation}

A calculation of agent $i$ should be started from the last agent in his scope, an agent $i+p$. This agent $i+p$ makes his decision based on his observable history from the point of agent $i$ view. The best response expression describes it.

$$
x_{i+p}=B R\left(A-\sum_{k=i+p-h}^{i+p-1} x_{k}\right)
$$

Then, we should calculate response of the next agent that is agent $i+p-1$. We have for this agent the following expression

$$
x_{i+p-1}=B R\left(A-\sum_{k=i+p-h-1}^{i+p-2} x_{k}-x_{i+p}\right)
$$

and then replace the decision of the agent $i+p$ by its expression from previous calculations

$$
x_{i+p-1}=B R\left(A-\sum_{k=i+p-h-1}^{i+p-2} x_{k}-B R\left(A-\sum_{k=i+p-h}^{i+p-1} x_{k}\right)\right)
$$

\section{Periodic equilibrium}

We are interested in periodic quasi-solutions because of three reasons. The first one is that computational models give us a periodic or almost periodic solution. The second reason is that periods are natural for economic dynamic. Moreover, the third reason is that the solutions are much more sophisticated than the general case.

The problem of combining is in the existence of a solution for periodic case only applicable far enough from the beginning and end of the 1 . So it might be that this periodic solution cannot be consistent with the other two cases. It might be a domain of future investigations. However, in any case, we can consider this research as an investigation of periodic solutions if they exist and when they arise due to two 
external out of model interventions. Since economic cycles are typical, we suggest this argument as reasonable. optimal

The following expressions are solutions for two periodic cases, with and without predictions. These expressions shows the utility of the Principal if every agent thinks that he is a last producer on the market

$$
F_{0}=\frac{k T s A}{(m+1+k T s) k}
$$

and the following is the utility of the Principal if every agent thinks that next agent is a last producer on the market

$$
F_{1}=\frac{(1-s) A T}{((k+m)(1-s) T-s)},
$$

where $T$ is a period, and $s$ is a constant that depends on type $r$ of the agents.

\subsection{Prediction zero}

Derivatives and change of the total market production by parameters. These derivates leads to explicit expression for the result of optimal control if the resource is very small, or even infinitely small as relaxation and approximation.

$$
\begin{gathered}
\sum_{j} x_{j}^{*}=\frac{\sum_{i} \frac{r_{i}}{2\left(r_{i}+1\right)} A}{m+1+k \sum_{i} \frac{r_{i}}{2\left(r_{i}+1\right)}}=\left(1-\frac{m+1}{m+1+k \sum_{i} \frac{r_{i}}{2\left(r_{i}+1\right)}}\right) \frac{A}{k} \\
\sum_{j} x_{j}^{*}=\left(1-\frac{m+1}{m+1+\frac{k T r}{2(r+1)}}\right) \frac{A}{k}
\end{gathered}
$$

Let

$$
\begin{gathered}
s=\frac{r}{2(r+1)} \\
\sum_{j} x_{j}^{*}=\left(1-\frac{m+1}{m+1+k T s}\right) \frac{A}{k}=\frac{k T s}{m+1+k T s} \frac{A}{k}
\end{gathered}
$$

Let we are given small resource which is enough to change only $\mathrm{m}, \mathrm{T}, \mathrm{k}$, or $\mathrm{s}$. We can predict how utility will change if we increase only one these parameters and only by 1 .

$$
\begin{gathered}
\Delta F_{0}^{m}=\frac{-\left(A(-1+s)^{2} T^{2}\right)}{((s-(1+k+m) T+(1+k+m) s T)} \times \\
\frac{1}{(-(k+m) T+s(1+k T+m T)))} \\
\Delta F_{0}^{T}=\frac{A(1+m) s}{(1+m+k s T)(1+m+k s(1+T))}
\end{gathered}
$$


Equilibrium in Generalized Stackelberg Game with Arbitrary Memory

$$
\begin{gathered}
\Delta F_{0}^{k}=-\frac{A s^{2} T^{2}}{(1+m+k s T)(1+m+(1+k) s T)} \\
\Delta F_{0}^{s}=\frac{A(1+m) T}{(1+m+k s T)(1+m+k(1+s) T)} \\
\Delta F_{0}^{s}=\frac{A(1+m) T}{(1+m+k s T)(1+m+k(1+s) T)} \\
\Delta F_{0}=\max \left(\Delta F_{0}^{m}, \Delta F_{0}^{k}, \Delta F_{0}^{T}, \Delta F_{0}^{s}\right) R
\end{gathered}
$$

Let we are given small resource again which is enough to change only $\mathrm{m}, \mathrm{T}, \mathrm{k}$, or s. We can predict how utility will change if we increase only one these parameters. It is applicable for large value of parameters, so we can approximate them as continuous parameters.

$$
\begin{gathered}
\frac{\partial F_{0}}{\partial m}=-\frac{A s T}{(1+m+k s T)^{2}} \\
\frac{\partial F_{0}}{\partial k}=-\frac{A s^{2} T^{2}}{(1+m+k s T)^{2}} \\
\frac{\partial F_{0}}{\partial T}=-\frac{A k s^{2} T}{(1+m+k s T)^{2}}+\frac{A s}{1+m+k s T} \\
\frac{\partial F_{0}}{\partial s}=-\frac{A k s T^{2}}{(1+m+k s T)^{2}}+\frac{A T}{1+m+k s T} \\
D F_{0}=\max \left(\frac{\partial F_{0}}{\partial m}, \frac{\partial F_{0}}{\partial k}, \frac{\partial F_{0}}{\partial T}, \frac{\partial F_{0}}{\partial s}\right) R
\end{gathered}
$$

\subsection{Prediction one}

$$
\begin{gathered}
\sum_{j=i}^{i+T} x_{j}=\frac{1-\frac{r}{2(r+1)}}{(k+m)\left(1-\frac{r}{2(r+1)}\right) T-\frac{r}{2(r+1)}} A T . \\
\sum_{j=i}^{i+T} x_{j}=\frac{1-s}{(k+m)(1-s) T-s} A T .
\end{gathered}
$$

Let we are given small resource which is enough to change only $\mathrm{m}, \mathrm{T}, \mathrm{k}$, or s. We can predict how utility will change if we increase only one these parameters and only by 1 .

$$
\begin{gathered}
\Delta F_{1}^{m}=-\frac{\left(A(-1+s)^{2} T^{2}\right)}{((s-(1+k+m) T+(1+k+m) s T))} \times \\
\frac{1}{(-(k+m) T+s(1+k T+m T)))}
\end{gathered}
$$




$$
\begin{gathered}
\Delta F_{1}^{T}=\frac{A(-1+s) s}{((s+k(-1+s)(1+T)+m(-1+s)(1+T)))} \times \\
\frac{1}{(-(k+m) T+s(1+k T+m T)))} \\
\Delta F_{1}^{k}=-\frac{A(-1+s)^{2} T^{2}}{((s-(1+k+m) T+(1+k+m) s T))} \times \\
\frac{1}{(-(k+m) T+s(1+k T+m T)))} \\
\frac{\Delta F_{1}^{s}=\frac{A T}{(1+s+k s T+m s T)(-(k+m) T+s(1+k T+m T))}}{\Delta F_{1}=\max \left(\Delta F_{1}^{m}, \Delta F_{1}^{k}, \Delta F_{1}^{T}, \Delta F_{1}^{s}\right) R}
\end{gathered}
$$

Let we are given small resource again which is enough to change only $\mathrm{m}, \mathrm{T}, \mathrm{k}$, or s. We can predict how utility will change if we increase only one these parameters. It is applicable for large value of parameters, so we can approximate them as continuous parameters.

$$
\begin{gathered}
\frac{\partial F_{1}}{\partial m}=-\frac{A(1-s)^{2} T^{2}}{(-s+(k+m)(1-s) T)^{2}} \\
\frac{\partial F_{1}}{\partial k}=-\frac{A(1-s)^{2} T^{2}}{(-s+(k+m)(1-s) T)^{2}} \\
\frac{\partial F_{1}}{\partial T}=-\frac{A(k+m)(1-s)^{2} T}{(-s+(k+m)(1-s) T)^{2}}+\frac{A(1-s)}{-s+(k+m)(1-s) T} \\
\frac{\partial F_{1}}{\partial s}=-\frac{A(1-s) T(-1-(k+m) T)}{(-s+(k+m)(1-s) T)^{2}}-\frac{A T}{-s+(k+m)(1-s) T} \\
D F_{1}=\max \left(\frac{\partial F_{1}}{\partial m}, \frac{\partial F_{1}}{\partial k}, \frac{\partial F_{1}}{\partial T}, \frac{\partial F_{1}}{\partial s}\right) R
\end{gathered}
$$

\section{Computational modelling}

This section provides numerical example for the given $h$ - memory and horizon of prediction $p$. First $h$ values are given and others are calculated based on exact formula of non periodic equilibrium. We have developed a software on Wolfram Research notebook to setup a system of equations and then solve them. Though they give an analytical solution, this solution is sophisticated thus we provide here only numerical results for different players types.

We have tried to investigate oscillations. The first agent make a nonzero move, then $h-1$ agents make zero moves. We investigated if we see oscillation as we had to $p=0$ and $p=1$ in our previous investigations, bifurcation, or there will be smooth plots. The figure shows that the small peaks in the beginning and that plats become more and more smooth with time. This effect does not depend on the type of agents $r$. Each line in the figure shows dynamics for the specific value of type $r$. 


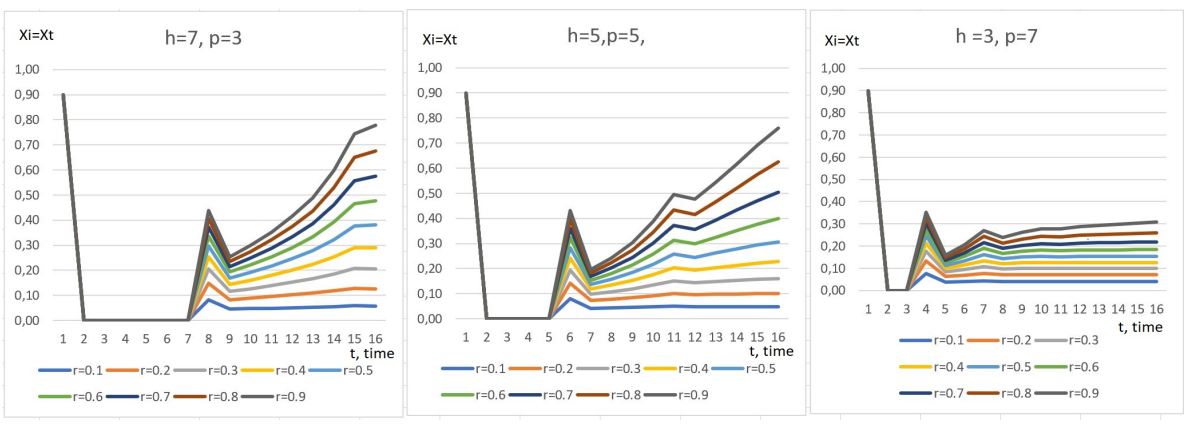

Fig. 6. Computational modelling of dynamics. Horizontal axis is time which is also an index of an agent. The vertical axis is production of agents with given index. Parameter $h$ is number of previous actions which are taken by an agent, and $p$ is planning horizon. Parameter $p$ shows how far is the last move in this game. A sum $p+h$ shows how many agents an agent $i$ see as his opponent if $i$ is far enough from the beginning.

Another property is that the more an agent pay attention to prediction rather than history, the faster the production growth. We do not know details of this process in general, but it can be formulated like "if people pay more attention to the future and less attention to the history, their production increases." We do not think the principle might be applied to any situation, but we have a hypothesis that works for our model.

\section{Discussion}

This paper suggested and investigated a model of generation change for Cournot competition with limited predictions and memory. We hope that this model might be helpful to model nuclear energy markets, paper-books markets, oil and gas markets, bitcoins, goods with an extended lifecycle. There are two main reasons for applications. The first reason is predictions. Some market lives longer than they are expected. People thought that oil and gas had vanished from energy markets, and VR and electronic books have eliminated paper books, but these markets still exist. Other people believe that bitcoins is a temporal thing, but this kind of money is still developing. The second reason is errors about the influence of the produced goods or services. People might think that produced electric cars or paper books will not be of any value soon, and there is no need to reduce market volume by producing volumes of these goods in the predictions. However, surprisingly indeed, people might use it like some people still use Windows 95 or Fortran. The key reason for these effects is epistemic effects, so our model also developed the epistemic model. We make some even more general results in epistemic modelling by generalizing a theory of mind model. This generalized model might be helpful for this model of market evolution and many models of partial awareness models or human-robot interactions. We hope that this model will be in computer science for epistemic modelling.

\section{References}

Rapoport, A. (1997). Order of play in strategically equivalent games in extensive form. International Journal of Game Theory, 26(1), 113-136. 
Abele, S., Herbert B., and K.-M. Ehrhart (2004). Social information processing in strategic decision-making: why timing matters. Organizational Behavior and Human Decision Processes, 93(1), 28-46.

Huck, S., and Müller, W. (2000). Perfect versus imperfect observability - an experimental test of Bagwell's result. Games and Economic Behavior, 31(2), 174-190.

Spiliotopoulou, E., Donohue, K. L., and Gurbuz, M. C. (2019). Do Allocation Mechanisms Drive Strategic Ordering? The Case of Integrated Distribution Systems. SSRN Electronic Journal, DOI: 10.2139/ssrn.3374813

Aumann, R. J. (1999). Interactive epistemology I: Knowledge. International Journal of Game Theory, 28(3), 263-300.

Fedyanin, D. N. (2018). An example of Reflexive Analysis of a game in normal form. In: Petrosyan L., Mazalov V., Zenkevich N. (eds) Frontiers of Dynamic Games. Static \& Dynamic Game Theory: Foundations \& Applications. Birkhauser, Cham, pp. 1-11 https://doi.org/10.1007/978-3-030-23699-1 1

Novikov, D., Chkhartishvili, A. (2014). Reflexion Control: Mathematical models. Series: Communications in Cybernetics, Systems Science and Engineering (Book 5). London: CRC Press, 298 p.

Byom, L.J., and Mutlu, B. (2013). Theory of Mind: Mechanisms, Methods, and New Directions. Frontiers in human neuroscience, 7, Article 413, https://doi.org/10.3389/fnhum.2013.00413

Wimmer, H., and Perner, J. (1983). Beliefs about beliefs: Representation and constraining function of wrong beliefs in young children's understanding of deception. Cognition, 13(1), 103-128.

Allaz, B., and J.-L. Vila (1993). Cournot competition, forward markets and efficiency. Journal of Economic theory, 59(1), 1-16.

Li, T., Sethi, S. P. (2017). A review of dynamic Stackelberg game models. Discrete \& Continuous Dynamical Systems-B, 22(1), 125-159. 